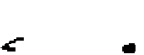

SLAC/AP- 51

JUL

DE86 013386

SLAC/AP - BI

April 1986

(AP)

\title{
SOME PROBLEMS ON RF BREAKDOWN IN ROOM TEMPERATURE ACCELERATOR STRUCTURE, A POSSIBLE CRITERION*
}

\author{
J. W. WAFG \\ Stanford Linear Aceleralor Center \\ Exanford Univernity, Stanford, California, 8/905 \\ DISCLATMER
}

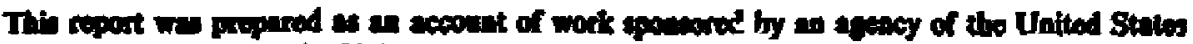

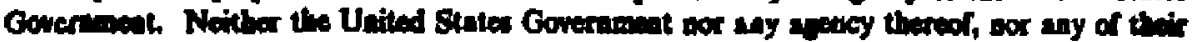

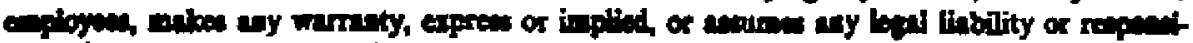

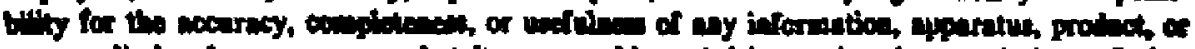

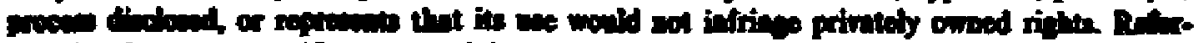

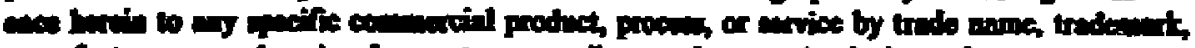

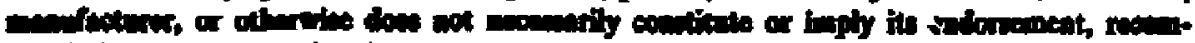

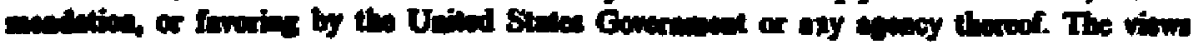

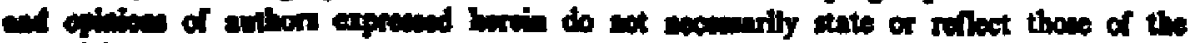

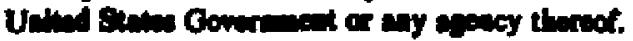

- Wort anppoted by the Deportment of Enary, coutract DE - ACO3 - 76\$F00515.

BISTRBOUTHO OF THIS DOCUMENT IS UMLAMITED 

RF field can be expressed as

$$
m \frac{d^{2} x}{d t^{2}}=e E_{0} \sin (\omega t+\phi)
$$

where $m$ and $e$ are the mass and charge of the particle, $E_{0}$ and $\omega$ are the peak field strength and angular frequency of the RF field. By solving the equation (1), the maximum energy gain $\varepsilon_{\max }$ is

$$
\varepsilon_{m a x}=\frac{2 t^{2}}{m}\left(\frac{E_{0}}{\omega}\right)^{2}
$$

For example, at $2856 \mathrm{MFz}$ and $E_{0}=300 \mathrm{MV} / \mathrm{m}$ the maxinum energy gain of a hydrogen ion is only $0.05 \mathrm{MeV}$. For other types of ions and for more arbitrary starting phases. $\varepsilon_{\text {mas }}$ would be much less. Thus, ion bombardment can almost be ignored. The electron multipacting can be suppressed if the peak electrical Geld $E_{0}$ satiofes the following condition: ${ }^{7}$

$$
E_{0}>\frac{m \omega^{2} d}{2 e}
$$

where $d$ in the cavity length. Actually the field gradients, that we are interested in are much higher than those shown in (3) above.

In this note we focus on the breakdown-initiating mechanism due to "cold" field electron emission occurring at isolated sites on broad-area cavity surfaces, where the field is locally enhanced. The influences of an alternating field and transition time of tunneling are taken into account. Finally the thermal instability resulting in vacuum voltage breakdown is hypothesized to derive a new criterion for room-temperature accelerator strueture. 


\section{Kilpatrick Criterion; It's Derivation and Availability to Accelerator}

In 1957, Kilpatrick stmmarized several investigators' experiences, data and theories. Considering the probability of field-emitted electrons together with the linear dependence of secondary-electron emission upon ion energy, the following equation was given to specify an upper limit for no vacuum sparking:

$$
W E^{2} \exp \left(-1.7 \times 10^{5} / E\right)=1.8 \times 10^{14}
$$

where $W$ is the maximum possible ionic energy in electron volt, and $E$ is the electric cathode gradient in $V / c m$. The relationship of $W \sim E$ is presented graphically as Fig. 1.

For RF the maximum possible ionic energy is related to transit-time and phasing effects. Kilpatrick studied the case of parallel plates electrodes with gap g. Figure 2 shows how $\boldsymbol{W}$ depends on RF voltage $V$ with frequency $f$. The quantity $V^{*}$ is defined by

$$
V^{*}(g / \lambda)^{2}\left(m_{0} c^{2} / \pi g\right)
$$

where $\lambda=\lambda / 2 \pi$ and $q$ is the electron charge, $M_{0}$ is the mass of atomic hydrogen. The threshold vacuum sparking data for $R F$ are in region $V / V^{*} \leq 1$. The slope in this region from 5 ig. 2 is approximately 0.61 , then we have

$$
W=\frac{0.153 q E^{2}}{M_{0} \pi f^{2}}
$$

By substituting $W$ of Eq. (6) into Eq. (4), the relationship of RF frequency in $\mathrm{MHz}$ and maximum electrical field in $\mathrm{MV} / \mathrm{m}$ is built as follows:

$$
f=1.64 E^{2} \exp \left(-\frac{8.5}{E}\right)
$$

From the derivation of the above formulae, they were not intended to be a criterion for RF breakdown in accelerators, which have varieties of cavity shapes. 
Also the modern vacuum equipments cavity surface machining and processing techniques are much improved compared to those of thirty years ago. No doubt further study is necessary.

\section{Field Electron Emission from Metal}

Following Sommerfeld, Fowler and Nordheim formulated the quantum mechanical tunneling of conduction electrons through the modificd potential barrier at the enurfnce of metal in an applied electric feld.,9 the one-dimensional barrier is illustrated in Fig, 3. The effective potential energy $V(x)$ is

$$
V(x)=\left\{\begin{array}{ll}
-W_{0} & \text { where } x<0 \\
-e E x-\frac{e^{3}}{4 x} & \text { where } x>0
\end{array},\right.
$$

the term $-\frac{e^{2}}{d x}$ is the contribution of the interaction with image cliarge. The tedious mathematical treatments can be outlined as follows. It is assumed that the conduction electrons in metal form a gas of free particles, which have FermiDirac statistics. The number of electrons within volume $v$ and with momenta within the indicated range $d P_{x} d P_{y} d P_{z}$ can be expressed by

$$
d n=\frac{2 v}{h^{3}} \frac{d P_{x} d P_{y} d P_{x}}{\sum^{c}+1}+1
$$

where $h$ is Planek's constant, $k$ is Boltzmann's constant, $s$ is cailed Fermi energy and $T$ is temperature. The flux of electrons that have normal energy components between $W_{x}$ and $W_{x}+d W_{x}$ and incident upon the barrier within the metal is $N\left(W_{x}\right) d W_{x} . N\left(W_{x}\right)$ is supply function which can be found by integrating Eq. (9) over all $P_{y}$ and $P_{z}$. The probability of penetration of the barrier $D\left(W_{x}\right)$ is callent the tranmission coefficient. By WKB approximation, we ras have the colution for the time-independent Schrödinger equation:

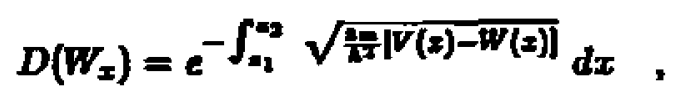

where $x_{1}$ and $x_{2}$ are the zeros of the radicand. The total number of electrons that turnel through the barrier is obtained by integrating the product of these 
functions $N\left(W_{x}\right) D\left(W_{x}\right) d W_{x}$ over all energies. Ordinarily the lowest energy $-W_{0}$ is far below the Fermi energy. By putting the lower limit at $-\infty$, the field emission current density is

$$
j F=e \int_{-\infty}^{\infty} D\left(W_{x}\right) N\left(W_{x}\right) d W_{z}
$$

For low temperature $\left(T \leq 300^{\circ} \mathrm{K}\right)$ it had the form:

$$
j F=\frac{1.54 \times 10^{-6} E^{2}}{\phi t^{2}(y)} \exp \left[\frac{-6.83 \times 10^{9} \phi^{1.5} v(y)}{E}\right]\left(\mathrm{Amp} / \mathrm{cm}^{2}\right)
$$

where $E$ is the surface electrical field in $\mathrm{V} / \mathrm{m}$, $\phi$ is the work function of the emitting material in $\mathrm{eV}, t(y)$ and $v(y)$ are tabulated dinoensionless elliptic functions, where $y=3.79 \times 10^{-5} E^{0.5} / \phi$ and $v(y)=0.956-1.062 y^{2}$. Substituting them into (12) and let $t(y) \approx 1$. The Fowler-Nordheim equation has the following form:

$$
j F=\frac{1.54 \times 10^{-6} \times 10^{4.524^{-03} E^{2}}}{\phi} \exp \left(-\frac{6.53 \times 10^{9} \phi^{1.5}}{E}\right)\left(\mathrm{Amp} / \mathrm{cm}^{2}\right) .
$$

As a practical consideration we introduce ideas of localized emission sites and enhancement factors. Any metal surface in accelerator cavities is far from planar and clean, there are large variations in the microscopic surface field. Local enhanced field would be created on some protruding microstructures, e.g. microprotrusions. The idealized microprotrusion geometries can be ellipsoid cylinders, needle-like shapes or other forms. By convergence, the microscopic field $E$ is geometrically enhanced to a higher microseopic value $E_{m}$ at the tip of the protrusion:

$$
E_{m}=\beta E
$$

The ratio of $E_{m}$ and $E$ is defined as the field-enhancement factor. Which depesds on the geometries and dimensions of a given protrusion. 
The external measurable parameter is field emissions current $I_{F}$ instead of current density $j_{F}$. By introducing the emitting area $A_{e}, \mathrm{Eq}$. (13) can be written in the following form: ${ }^{10}$

$$
\frac{d\left(\log _{10} I_{F} / E^{2}\right)}{d(1 / E)}=-\frac{2.84 \times 10^{9} \phi^{1.5}}{\beta}
$$

This is the slope for a Fowler-Nordheim plot of $\log _{10} I_{F} / E^{2}$ versus $1 / E$, and the intercept is

$$
\log _{10}\left(I_{F} / E^{2}\right)_{E \rightarrow \infty}=\log _{10}\left[\frac{1.54 \times 10^{-6} A_{\epsilon} \beta^{2} 10^{1.52 \phi^{-0.4}}}{\phi}\right] \text {. }
$$

Numerical calculation of the effects of random emitter arrays on measured FowlerNordheim plots based on the total field emission current have been carried out 11,12 and these indicate that in general, a straight line fit car be expected, the slope of which approximates the $\beta$ value of the sharpest emitter. The determined emitting area corresponds to a value somewhat less than that associated with a value computed for all the emitters.

There are more hypotheses proposed to explain the enhanced emissions: oxide films and associated trapped positive surface charges, partially embedded nonmetallic impurity particles, absorbed particles on metal surface possessing a special band structure to enhance tunneling, etc. Some of these hypotheses predict that the field emission current would have the characteristic Fowler-Nordheim form while others predict a different relationship. But under clean condition the pre-brealdown current has been established as obeying the Fowler-Nordheim equation according to many experimental results. It is acceptable to use effective $\boldsymbol{\beta}$ as a judgment for the field enhancement degree of a certain accelerator cavity surface. This enhancement can be due to electrical field onhancement by microprotrusions or due to current increase based on other mechanisms mentioned above. Still the Eq. (15) is a very important theoretical basis for experimental measurements of $\beta$ value. 


\section{Field Electron Emission for Alternating Field}

We suppose the surface field in an accelerator cavity is $E=E_{0} \sin \omega t$ and also assume that the field emission happens at a very short period of time compared to the RF periad $T$. The average field emission current can be obtained by time-averaging:

$$
\begin{aligned}
& \bar{I}_{F}=\frac{1}{T} \int_{0}^{T} I_{F}(t) d t
\end{aligned}
$$

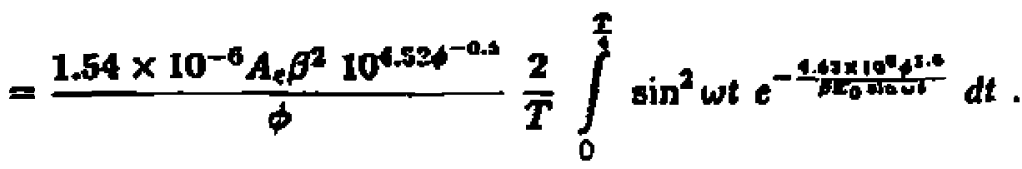

We integrate by approximation as Ref. 13 , let

$$
S(\mu)=\frac{2}{T} \int_{0}^{\frac{T}{T}} \sin ^{2} \omega t e^{-\frac{T}{15}} d t
$$

with

$$
\mu=\frac{e .53 \times 10^{9} \phi^{1.5}}{\beta E_{0}}
$$

and change the variables: $\sin \omega t=\frac{1}{2}$, then

$$
\begin{aligned}
S(\mu) & =\frac{1}{\pi} \int_{1}^{\infty} \frac{e^{\mu \pi}}{x^{3} \sqrt{x^{2}-1}} d x \\
& =\frac{1}{\pi} \int_{1}^{\infty} R(x) \frac{e^{\mu x}}{\sqrt{x-1}} d x
\end{aligned}
$$

with

$$
R(x)=\frac{1}{x^{3} \sqrt{x+1}}
$$




$$
S(\mu) \approx \frac{1}{\pi} \tilde{R}(\mu) \int_{1}^{\infty} \frac{e^{\mu x}}{\sqrt{x-1}} d x=\frac{\tilde{R}(\mu)}{\sqrt{\pi \mu}} e^{-\mu}
$$

$\tilde{R}(\mu)$ is a slowly varying function of $\mu$. For example, $\phi_{e u}=4.65 \mathrm{eV}, \beta E \sim 6 \times 10^{\circ}$ $\mathrm{MV} / \mathrm{m}, \mu \approx 11$, by using a computer the results of numerical integration show that corresponding to different conducting material and large variation of field strength, $\mu$ can change from 8 to 20 , but $R(\mu)$ only changes from 0.55 to 0.57 . So we can have the field-emission current for alternating field:

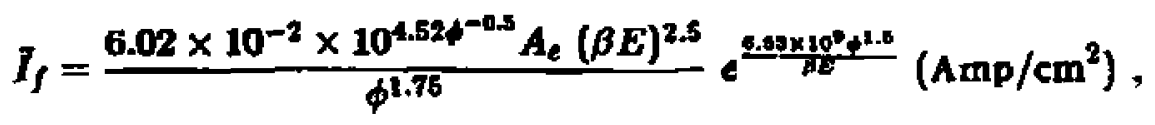

where $\phi$ is the work function in $\mathrm{eV}, E$ is the surface field in $\mathrm{V} / \mathrm{m} \beta$ is the enhancement factor and $\bar{I}_{F}$ is the average field emission current on an emitting area $A_{c}$ in $\mathbf{m}^{2}$.

Furthermore, we should consider the retardation of electrons in tunneling through a bartier. For quasi-stationary state single particle tunneling, there exists much discussion about this retardation or iunneling life time. ${ }^{14}$ The tunneling situation can be modeled as a time-dependent two-state system, in which the time-dependent basis states were taken to be localized states, $\psi_{\text {a }}$ and $\psi_{k}$ on each side of the barsier. When the perturbation $V$ added, the two states couple through the overlep of their exponential tails in the barrier region. The system, known to have been in the initial discrete state $s$ at $t=0$, will be in the unperturbed final cigenstate $k \neq 3$ at time $t$. The probability is then given by ${ }^{15}$

$$
\left|C_{k}(t)\right|^{2}=2\left|\left\langle\psi_{k}|V| \psi_{s}\right)\right|^{2} \frac{1-\cos \omega_{k, t} t}{\left(E_{k}-E_{s}\right)^{2}}
$$

wherc $E_{k}, E_{k}$ are energies for states $\psi_{n,} \psi_{k}$ and $\omega_{k \downarrow}=\left(E_{k}-E_{t}\right) / \hbar=\delta E / \hbar$. Let $D^{\prime}(t)=1 \rightarrow \cos \omega_{k, t} t=1-\cos \frac{\delta E}{\hbar} t$, after a time $\tau=\frac{\pi \AA}{2 \delta L}$, we have $D^{*}(\tau)=1$. It means that the electron would become a free electron. The $\delta E$ is proportional 
to $e^{-k w}, 16$ where $w$ is the barrier width and $k$ in the decay constant:

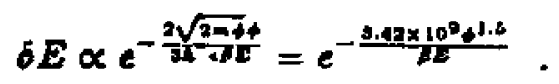

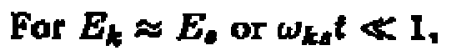

$$
D^{\prime}(t) \approx \frac{1}{2}\left(\frac{\pi t}{2 r}\right)^{2},
$$

i.c. the transition probability increases quadratically with time. In an actual RF field the tunneling is concentrating in the hat top near $E_{\text {max }}$, for instance, with an effective time for tunneling $\Delta t \approx 0.1 / f .^{\prime 7}$ By using Eqs. $(18,20)$

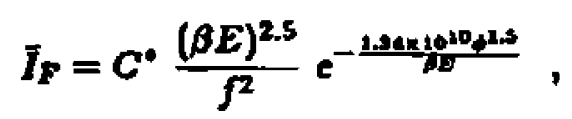

where $C^{*}$ is a constant.

For a certain structure the slope for a plot of $\log _{10} I_{F} / E^{2.5}$ versus $1 / E$ has the following form:

$$
\frac{d\left(\log _{10} J_{P} / E^{2.5}\right)}{d(1 / E)}=-\frac{5.8 \times 10^{9} \phi^{1.5}}{\beta}
$$

Therefore, this important parameter of $\boldsymbol{\beta}$ value can be obtained by performing field emission current measurements. 


\section{Breakdown Mechanisms and Criteria}

The very high field emiseion currents flowing through the mitroprotrusions may cause excessive resistive heating. Then the localized melting or vaporization processes the tip areas lead to the creation of a microplasma. This is the essentjal prerequisite for a breakdown event. ${ }^{10}$ Under good vacuum conditions this is the only origin of an ionizable medium. Several energy exchanges are involved by a high field emission current flowing into the tip of the ernitter, thermal cooling by heat conduction down the shank of the protrusion in to the "cold" reservoir provided by the bulk of the cavity body, thermal cooling by radiation losses from the aurface of the emitter (which plays an insignificant role). Also there exist internal heating or cooling, the so called Nottingham effect. This effect, which can cause heating or cooling, arises because of a difference between the average energy of emission and the average energy of the electrons replacing the emitted electrons. In order to simplify the problem, consider a thermafly steady-state solution for the truncated cone type of emitter as shown in Fig. 4. The difference betwe $n$ the top temperature $T_{r}$ and the base temperature $T_{b}$ of the emitter is ${ }^{18}$

$$
\Delta T=T_{r}-T_{b}=\left[\Omega(T) / 2 K \pi^{2}\right]\left(I_{F} / \alpha r\right)^{2}+\left(k T_{r} I_{F} / K \alpha r\right) \cos \pi P
$$

where $I_{F}$ is the total emission current, $\Omega(T)$ is the electrical resistivity and $K$ is the thermal conductivity of the emitter, $k$ is Boltzma $\eta^{\prime} 8$ constant, $P \approx 9.3 \times$ $10^{5} \phi^{0.5} T / E$. The first term of Eq. (22) is due to resistive heating, it increases rapidly with emission and temperature, the second term represents Nettingham effect. For less refractory metal such as copper, whose melting point is $\sim 1360^{\circ} \mathrm{K}$, Nottingham heating is the dominating factor determining the temperature of microprotrusions.

Let $T_{\mathrm{r}}$ equal melting temperature and reasonably to assume $\alpha r$ proportional to $1 / \beta$. According to the discussions above we can derive gun RF breakdown 
criterion as follows:

$$
f=C \beta^{1.75} E^{1.25} \exp \left(\frac{-6.68 \times 10^{9} \phi^{1.5}}{\beta E}\right)
$$

$C$ is a constant related to material characteristics such as work function, resistivity and thermal conductivity. The building materials for accelerator cavities and $\mathrm{RF}$ components usually are $\mathrm{Cu}, \mathrm{Al}$ or material coated with $\mathrm{Ag}$ or $\mathrm{Au}$, all of which have very common characteris tics. For SLAC well-RF processed cavities the measured $\beta=102$ and breakdown linit $E_{\text {max }}=300 \mathrm{MV} / \mathrm{m}$ were reached for $2856 \mathrm{MHz}$ RF field. A possible RF breakdown criterion then can be written as:

$$
f=6.55 \times 10^{-3} \beta^{1.75} E^{1.25} \exp \left(\frac{-6.68 \times 10^{9} \phi^{1.5}}{\rho E}\right)
$$

where $f$ is RF frequency in MHz. $E$ is surface electrical feld limit in $V / m$. $\phi$ is work function of cavity material in $\mathrm{eV}$, and $\beta$ is measured enhancement factor. For different $\beta$ value, a group of curves are plotted in Fig. 5.

The Eq. (25) and curves in Fig. 5 have very clear physicg meanings. For a certain RF frequency when RF processing was performed, the field emission current would be reduced gradually. Often $\beta$ value is getting smaller, especially in the beginning of the processing. The sparking or breakdown threshold can be increased. This progress corresponds to a horizontal dashed line in Fig. 5. According to the microwavescaling law, lower frequency accelerators have relatively larger cavities, and condeminational areas are larger. Many experimental data showed this situation. Thus we can explain why the lower frequency accelerators have lower RF breakdown thresholds.

\section{Acknowledgements}

The author gratefully acknowledges the valuable advicc and encouragement of Prof. G. A. Loew. Thanks are also due to Profs. P. B. Wilson, V. Nguyen-Tong and $R$. H. Miller for useful discussions. 


\section{References}

1. W. D. Kilpatrick, "Criterion for Vacuum Sparking Designed to Include Both RF and DC." UCRL_2321, Sept. 1953.

2. E. Tanabe, "Voltage Breakdown in S-Band Linear Accelerator Cavities," IEEE Trans. Nucl. Sci., NS-30, p. 3351, 1983.

3. E. Tanabe, "Breakdown in High-Gradient Accelerator Cavities," Proceedings of the 1984 Linear Accelerator Conference, Seeheim-Darmstadt, West Germany, p. 403, 1984.

4. J. W. Wang and G. A Loew, "Measurements of Ultimate Accelerating Gradients in the SLAC Disk-Loaded Strueture," IEEE Trans. Nucl. Sci., N532, p. 2915, 1985.

5. S. W. Williams et al., "Voltage Breakdown Testing for the Radio-Frequency Quedrupole Accelerator," Proceedings of 1979 Linear Accelerator Conference.

6. A. Gerhard et al., "RF Sparking Experiments," IEEE Trans, on Electrical Insulation," Vol. EI-20, No. 4, Aug. 1985.

T. M. S. Livingston and J. P. Blewett, "Particle Accelerators," p. 346, MeGrawHill Book Company, Inc. 1962.

8. R. H. Fowler and L. Nordheim, Proc. Roy. Soc. A119, 173-81, 1928.

9. R. H. Good and E. W. Müller, In "Handbuch der Physik" Springer Verlag, Berlin, 21, 176-231, 1956.

10. R. V. Iatham, "High Voltage Vacuum Insulation: The Physical Basis," Academic Press, 1981.

11. H. E. Tomasche and D. Alpert, "Fjeld Emission from a Muitiplicity of Emitters on a Broad-Area Cathode," J. Appl. Phys. 38, 881, 1967.

12. G. A. Farrall, "Numerical Analysis of Field Emission and Thermally Enhanced Emission from Broad-Area Electrode in Vacuum," J. Appl. Phys. 
41, 5631970.

13. H. Schopper, HI. Strube and L. Szecsi: "An Investigation of Field Emission in Superconducting RF Cavities by Dose Rate Measurements, ${ }^{n}$ KFKExtern, 3/68-6.

14. J. W. Gadzuk and A. A. Lucas, "Field-Emission Tails and Tunneling Lifetime," Phys. Rev. B7, 4770, 1973.

15. E. Merzbacher, "Quantum Mechanics," 2nd ed. John Wilcy \& Sons Inc. p. 476, 1970.

16. E. O. Kane, "Tunneling Phonomena In Solid," edited by E. Burstein and S. Lundquist, Plenum. New York, p. 9, 1969.

17. J. Halbritter, On Electron Emission Phenomena from Oxidized Metal Surfaces, Their Application to Electron Loading in Niobium High Frequency Cavities," KFK-Extern, 3/78-1.

18. L. W. Swanson, L. C. Crouser and F. M. Charbonnier, "Energy Exchanges Attending Field Electron Emission," Phys. Rev. Vol. 151, No. 1, 327, 1966. 


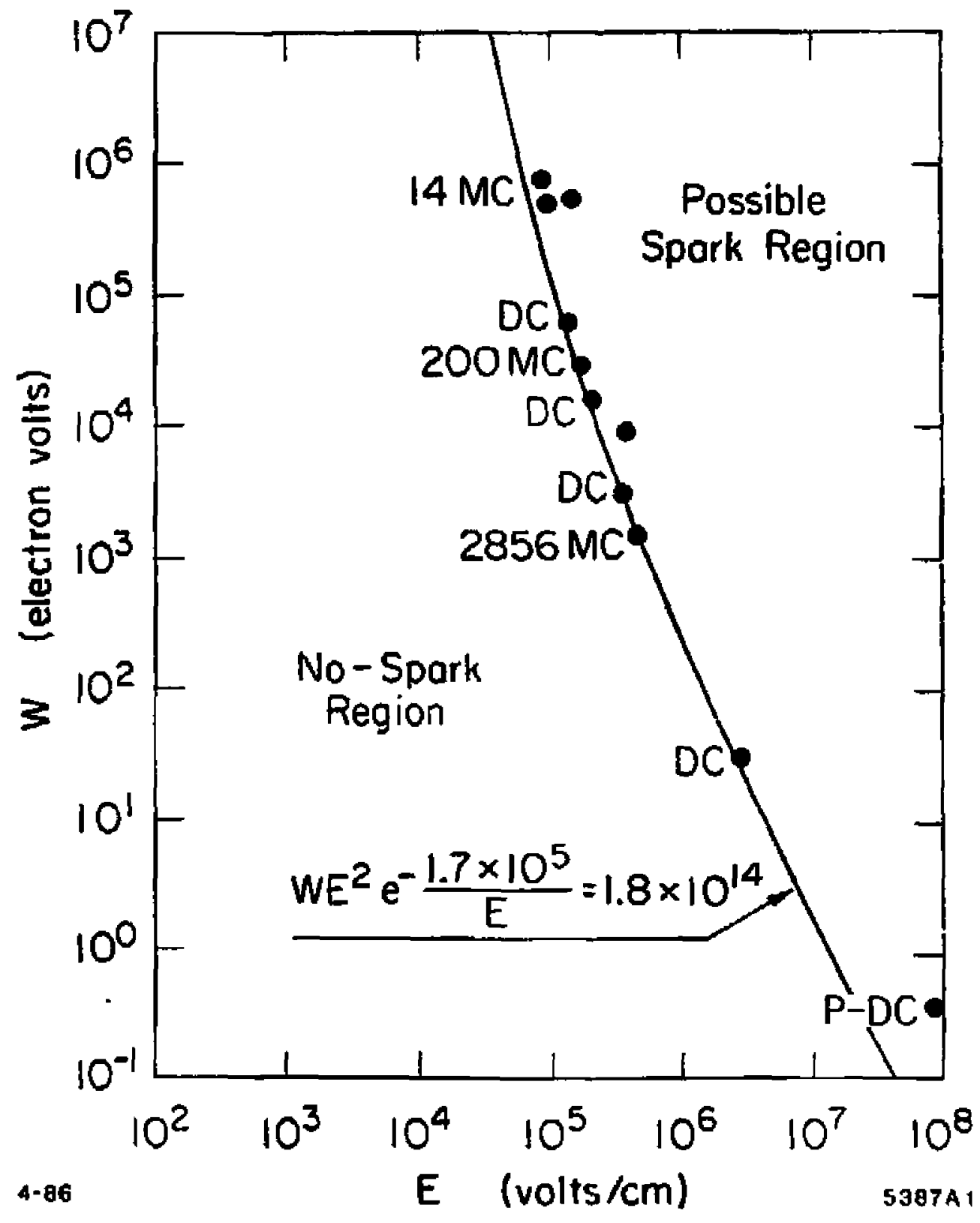

Fig. 1 : $W$ plotted against $E$, the cathode gradient. For DC, $W$ corresponds to the applied voltage; for RF, $W$ is a function of frequency and gap. (From Kilpatrick [1]). 


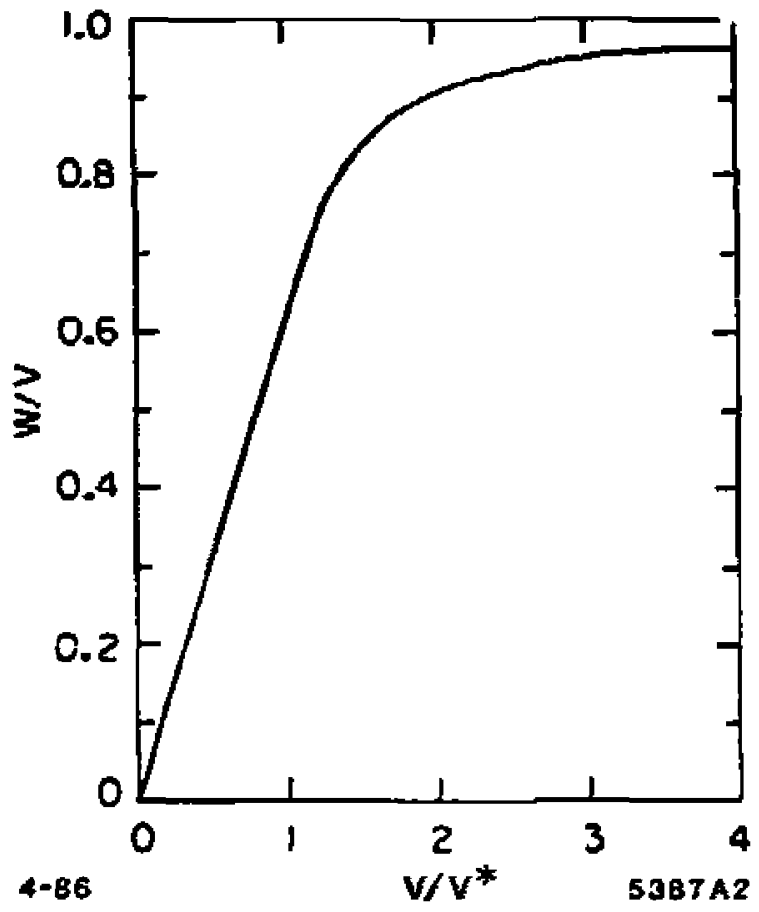

Fig. 2: Graph for ion transit-time correction. For plane parallel fields, $V^{*}=$ $(g / \hbar)^{2} M_{0} c^{2} / \pi q$. For computing the maximum cathode ion energy, where $\nabla \cdot E$ is not $\approx 0$, the calculation of $W$ or $V^{*}$ is without reference. (From Kilpatrick [1]). 


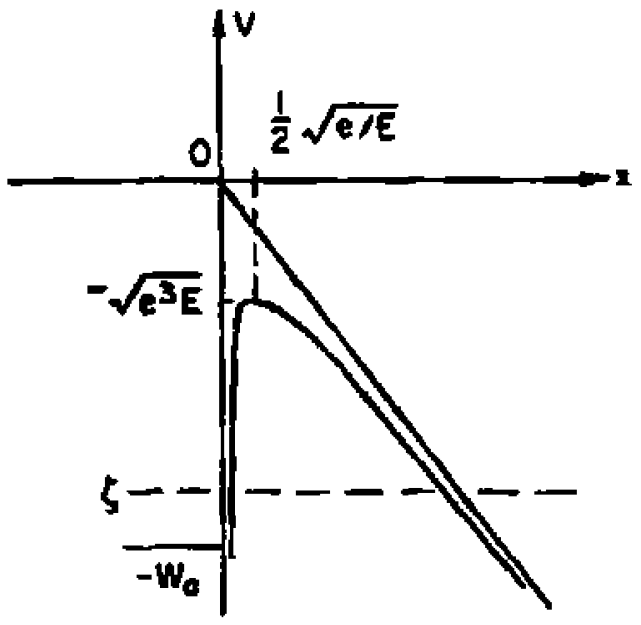

4-07

$5387 \times 3$

Fig. 3 : Potential energy diagram. 


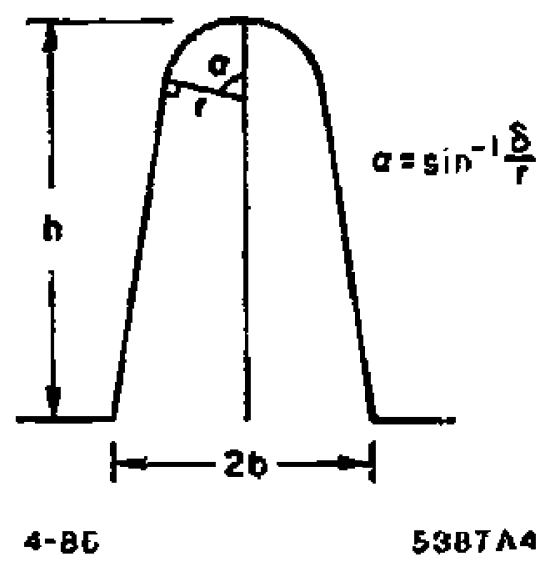

Fig. 4: An idealized field-emitting microprotrusion. 


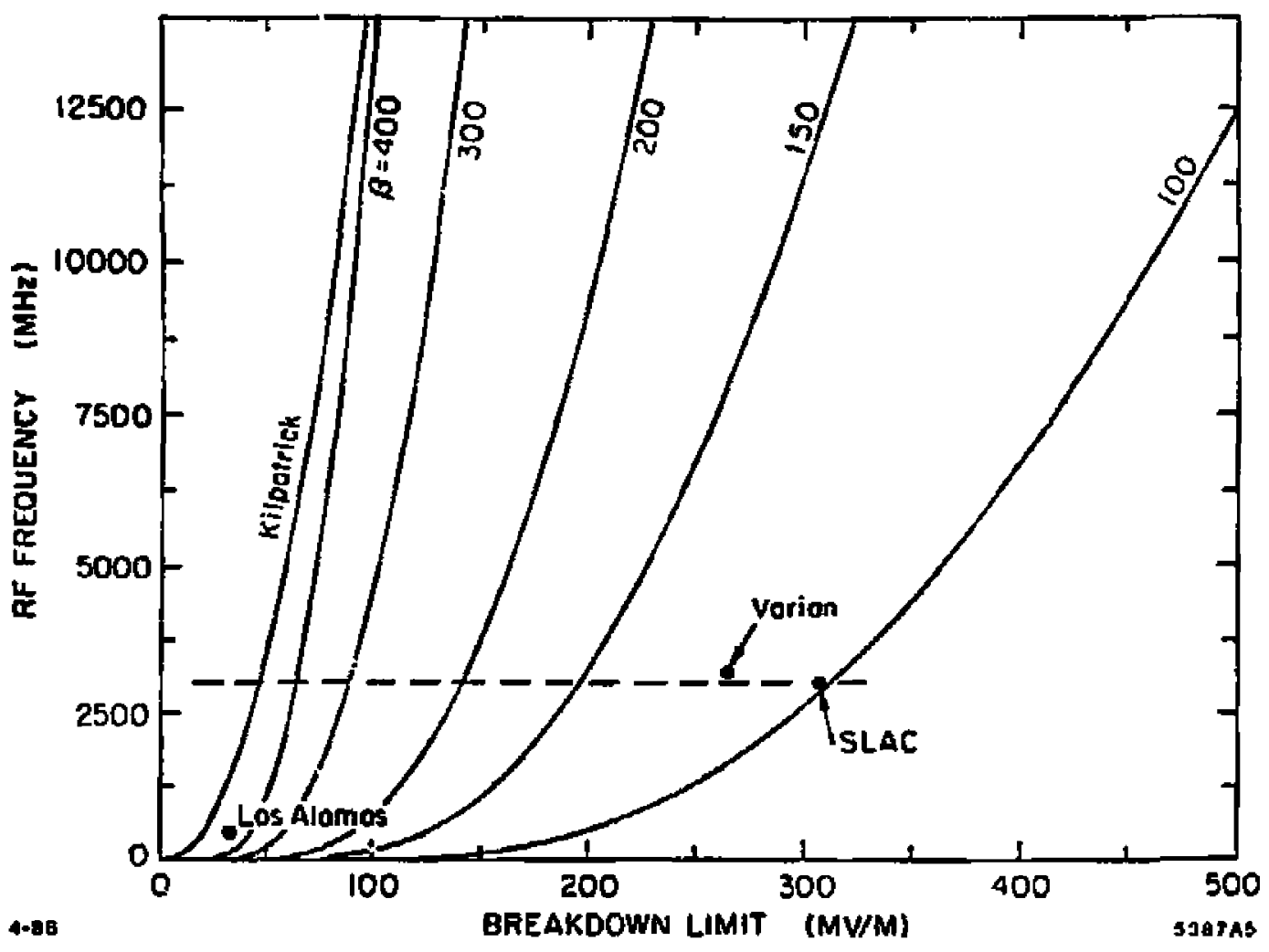

Fig. 5 : RF breakdown criteria and some experimental results. 